\title{
Geometrical Parameters Optimization of Suction Cups-Based Devices for Large-Sized Ceramic Slabs Handling
}

\author{
I. Campione ${ }^{1 *}$, M. Troncossi ${ }^{1}$, C. Maiello ${ }^{1}$, G. Lucisano ${ }^{2}$ \\ ${ }^{1}$ Interdepartmental Center for Applied Research on Advanced Applications in Mechanical \\ Engineering and Materials Technology, Alma Mater Studiorum University of Bologna. Via \\ Fontanelle 40, 47121 Forlì, Italy \\ ${ }^{2}$ Interdepartmental Center for Applied Research on Buildings and Construction, Alma Mater \\ Studiorum University of Bologna. Viale Risorgimento 2, 40136 Bologna, Italy \\ e-mail: ivo.campione@gmail.com \\ e-mail: marco.troncossi@unibo.it \\ e-mail: maiellocristian@libero.it \\ e-mail: giuseppelucisano@gmail.com \\ *corresponding author
}

\begin{abstract}
In the last decades, due to remarkable technological improvements in ceramics production cycles and materials, the manufacturing and the commercialization of large-sized ceramic slabs have risen. While some phases of the process are well defined, there are some routines that lack systematic procedures and rely uniquely on the internal know-how of the single companies. In particular, this paper focuses on the handling and transportation of large-sized ceramic slabs, which are, in the vast majority of cases, performed by suction cups-based gripping devices. The scope of this paper is to investigate how the type of suction cup pattern and its geometrical parameters affect slab stresses and deformations and to provide a guideline for the choice of the pattern, in order to cope with some critical aspects associated with wide surface and small thickness of the slabs. In particular, some of the most common large slab sizes and thicknesses available on the market are considered, and the most suitable suction cup patterns (and their geometrical parameters) for handling them are evaluated in order to minimize slab stresses and deformations.
\end{abstract}

Keywords: Transportation, handling, large-sized slabs, suction cups, ceramics, porcelain stoneware, finite element, ANSYS

\section{Introduction}

Porcelain stoneware is a very compact, hard and low-porous material, highly vitrified, hence with excellent mechanical and chemical properties. Some of its most outstanding properties are frost resistance (making it extremely suitable for outdoor flooring and wall cladding in cold climates) and high resistance to chemical substances and cleaning agents. Furthermore, it resists abrasion well and has a high ultimate strength, which makes it ideal for industrial areas and 
highly frequented spots. In addition, porcelain is very easy to clean and thus particularly fitting for places where hygiene is of prime importance.

In the last decade, due to outstanding developments in materials field, further improvements in ceramic quality have been made, concerning both functional properties and aesthetical appearance. Tucci et al. (2007) for instance, considered new body mixes for porcelain stoneware tiles to enhance mechanical characteristics, and Leonelli et al. (2001) investigated how to enhance the mechanical properties by using a micro-structural approach. These improvements in the ceramics properties have led to an increase and expansion of the ceramic tile market. Notably, one of the latest market trend goes towards large dimensions (from $60 \times 60 \mathrm{~cm}$ up to $160 \times 320 \mathrm{~cm}$, which is the current maximum dimension on the market) and a very low thickness of tiles, whose minimum value is $3 \mathrm{~mm}$ (Vivona et al. 2009, Morselli 2009). In order to produce large-sized ceramic slabs, innovative technological solutions are applied, involving a novel process that relies on a new approach to both shaping and thermal treatments (Raimondo et al. 2009, Gozzi et al. 2009).

The physical, chemical and mechanical properties of large-sized ceramic slabs conform to the top quality range of porcelain stoneware tiles, and a large size coupled with low thickness grants the slab a certain degree of flexibility, which can be enhanced by using ceramic fiberglass composites (Tasdamerici et al. 2009). These remarkable performances make largesized slabs suitable to be used for building and construction (flooring and ceiling, interior wall tiling, ventilated façades, tunnel coverings, insulating paneling), indoor furniture (table tops, doors) and support for photovoltaic ceramic panels. In Fig. 1, different slabs produced by a company are shown: it is possible to make a comparison between the standard sizes (from $20 \mathrm{x}$ $20 \mathrm{~cm}$ up to $75 \times 150 \mathrm{~cm}$ ) and the very large sizes, up to $160 \times 320 \mathrm{~cm}$ and with very low thicknesses (6 mm and $9 \mathrm{~mm}$ ). In Fig. 2 an indoor application is shown.
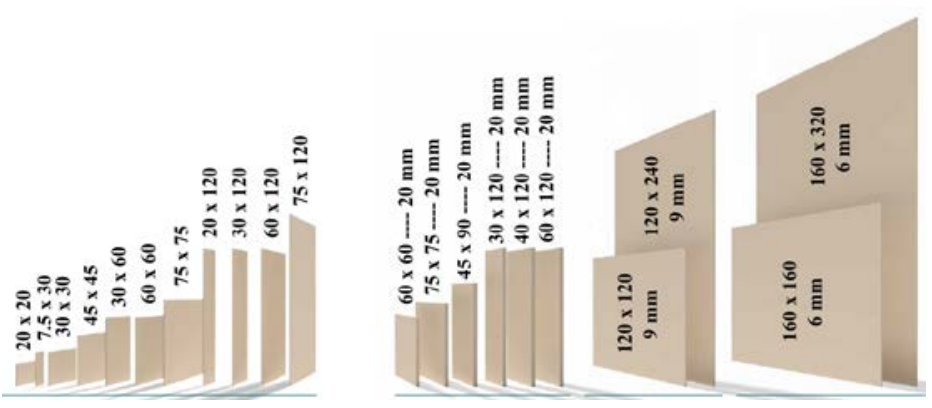

Fig. 1. Commercial slabs produced by a company (Caesar 2015). Starting from the left: the standard sizes, sizes with a greater thickness $(20 \mathrm{~mm})$, large-sized slabs 


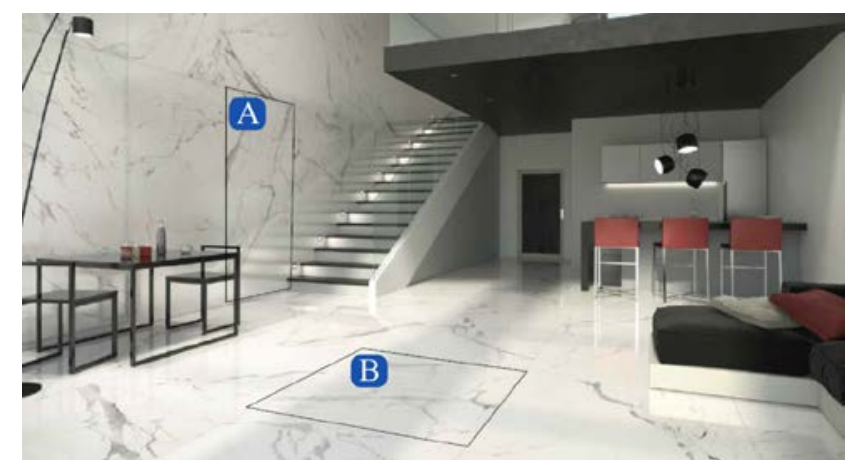

Fig. 2. Example of application of large-sized ceramic slabs in indoor applications. A: $120 \times 240$ cm; B: 120 x 120 cm. (Caesar 2015)

Despite the global significance of porcelain tile manufacture and the technological advances, the scientific activity in porcelain tile's almost 30-year existence does not match the importance of the business (Sánchez et al. 2009). This remark applies perfectly when considering large-sized slabs: while the manufacturing process is basically already well defined, there are some aspects that still lack some proper scientific studies and standard operative procedures. In order to contribute to fill this gap, Fragassa, Pavlovic et al. (2014, 2015, 2016) examined the effects on the mechanical properties of the innovative application of pyroclastic deformation on the ceramic slabs, with the aim to provide new tools to approach the quality control in non- traditional ceramics.

One tricky phase, which still has not been the object of a proper analysis, is the handling and transportation of large-sized slabs that may be critical for several reasons. Indeed, considering the large dimensions and the reduced thickness, the handling and transportation have to be done in a specific way to avoid high stresses, mainly due to flexure. For simple transportation tasks, usually automated, common devices are roller and/or belt conveyors. However, situations in which slabs are manually handled or in general when their transportation involves more complex routes and movements, are very common, and specific gripping devices are needed to fulfill these tasks. By gathering data from different companies, one can find that the most popular gripping devices rely on suction cups (typically from 2 to 6), arranged in different patterns. Suction cups are very suitable for handling large slabs because they are able to provide the necessary amount of gripping force, they only need the upper slab surface to operate and they are practical and fairly economical. The suction cups are attached onto a handling frame (usually composed of steel bars), and can be moved back and forth along the bars axes and locked at the desired distance. A device with two suction cups is shown in Fig. 3, where the operating principle of the suction cups is also highlighted: the internal depressurization, monitored by a barometer, is usually obtained manually, by repeatedly pushing a proper designed cylinder in contact with the internal suction cup region. 

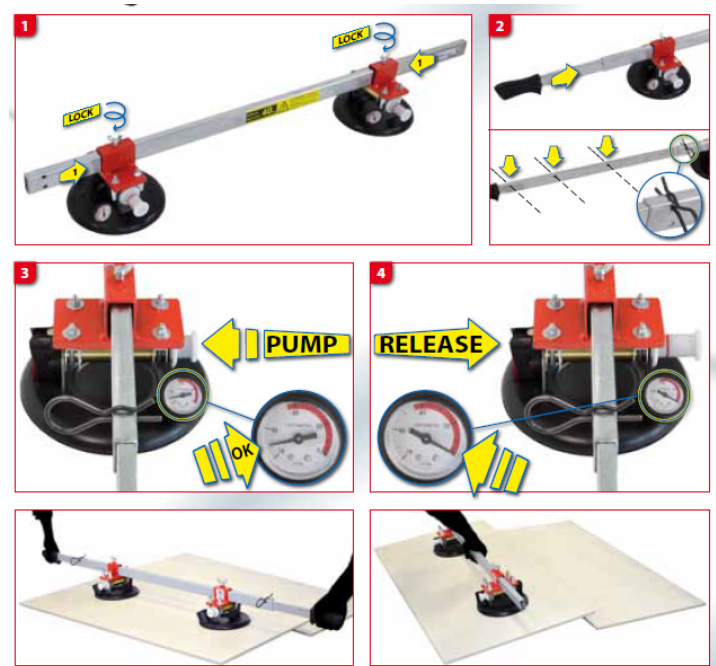

Fig. 3. Gripping device with two suction cups. 1: possibility to move the suction cups along the bar axis. 2: possibility to extend the bar length. 3 and 4: generation of the depressurization and release, respectively, by pushing proper parts (Brevetti Montolit).

In Fig. 4, two other typical suction cup gripping devices are shown in case of four (a) and six (b) suction cups. Figure 5 shows the slab handling operation, carried out manually by two workmen with the device of Fig. $4 \mathrm{a}$.
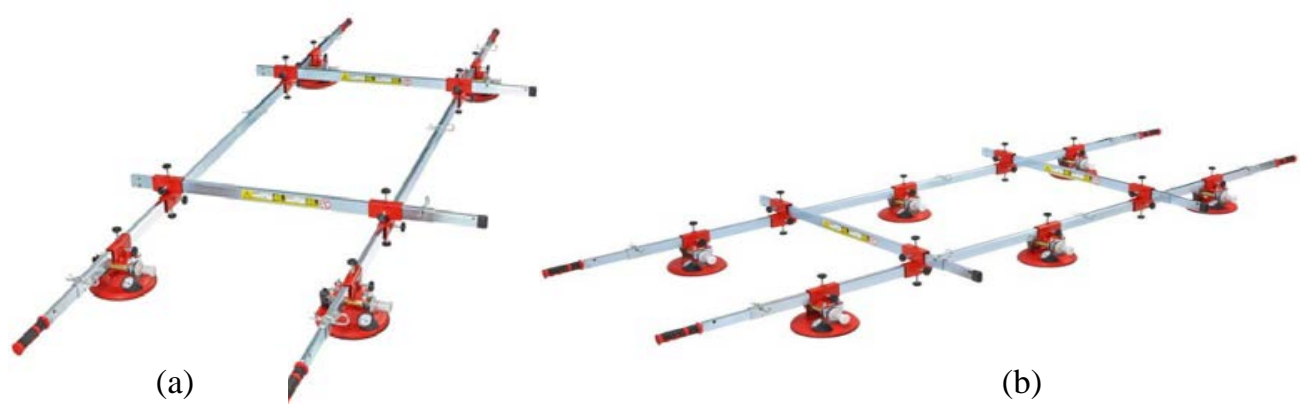

(b)

Fig. 4. Gripping device with four (a) and six (b) suction cups. (Brevetti Montolit) 


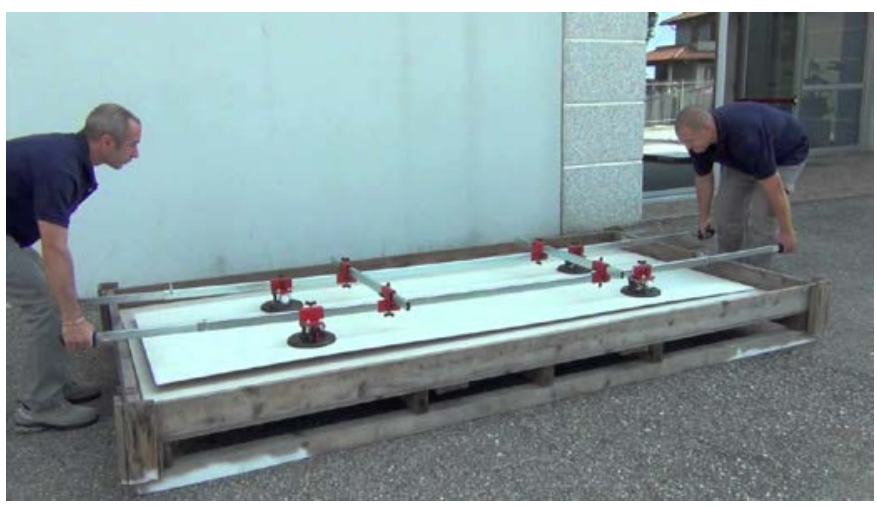

Fig. 5. Slab manually handled by two workmen

Even if several gripping devices are present on the market, the choice of the most suitable ones for specific handling operations of large-sized slabs is often not well justified and/or analyzed. Furthermore, for the evaluation of the proper suction cup distances along the bars, one relies exclusively on the expertise of the operators. To address these limitations, the influence of three of the most typical suction cup patterns on the slab stress distribution and deformation is analyzed, considering the most common sizes and thicknesses of the ceramic large-sized slabs on the market.

From a theoretical point of view, the problem is related to the thin plate theory (S. Timoshenko 1989), whose governing equation is the fourth-order partial differential equation often known as the biharmonic equation. The resolution methods depend on the boundary conditions, and even if various approaches are present in the literature for the case of differently constrained edges, methods able to deal with free boundary conditions and support conditions are rare, with some very recent exceptions (R. Li et al. 2016). For these reasons, a finite element approach was chosen and the software ANSYS Workbench was used. ANSYS Workbench is software commonly utilized for finite element modeling, and some examples can be found, for instance, in the work of Fragassa and Pavlovic (2016), concerning the viscoelasticity of ceramic tiles, or in the work of Tsamasphyros et al. (2009). One particular feature of ANSYS Workbench, which is exploited in this paper, is the possibility of doing parametrical analyses. An example of ANSYS parametrical analysis approach can be found in the paper of Wang et al. (2008), where a parametrical approach is taken for design optimization purpose.

Before running the simulations, various aspects of the problem were analyzed in order to define a proper working procedure. Then, the simulations were run following a specific plan and the results were analyzed and discussed. The final purpose of the presented investigation, concerning the influence of the pattern type and its geometrical parameters on the slab stresses and deformations, was the definition of a guideline aimed at helping the pattern choice in practical situations.

\section{Materials and Methods}

\subsection{Main Components}

Commercial suction cups used for this purpose have a nominal diameter of about $20 \mathrm{~cm}$, and are able to support about $20 \mathrm{~kg}$ each. 
With regard to the slabs, according to a market analysis, the following typical large sizes were taken into account:

\begin{tabular}{|c|c|}
\hline Type & Size $[\mathrm{mm}]$ \\
\hline 1 & $1200 \times 1200$ \\
\hline 2 & $1600 \times 1600$ \\
\hline 3 & $1200 \times 2400$ \\
\hline 4 & $1000 \times 3000$ \\
\hline 5 & $1600 \times 3200$ \\
\hline
\end{tabular}

Table 1. Most common commercial slab sizes

Since thickness is a very important parameter in evaluating the stress distribution, for each size the thickness was considered a variable from $3 \mathrm{~mm}$ to $15 \mathrm{~mm}$, a range that embraces all the most common thicknesses of the sizes listed in Table 1.

\subsection{Suction Cup Patterns}

Three suctions cup patterns were considered, the ones most easily supported by a light and simple steel structure, typically composed of a series of steel bars. The three patterns $A, B$ and $C$ are formed by 2, 4 and 6 suction cups, respectively (Fig. 6). Each pattern was considered symmetrical, due to the uniform stress distribution that they are able to guarantee. In addition, this choice made the theoretical investigation quite simple, since only a few parameters were needed to define the position of each suction cup.
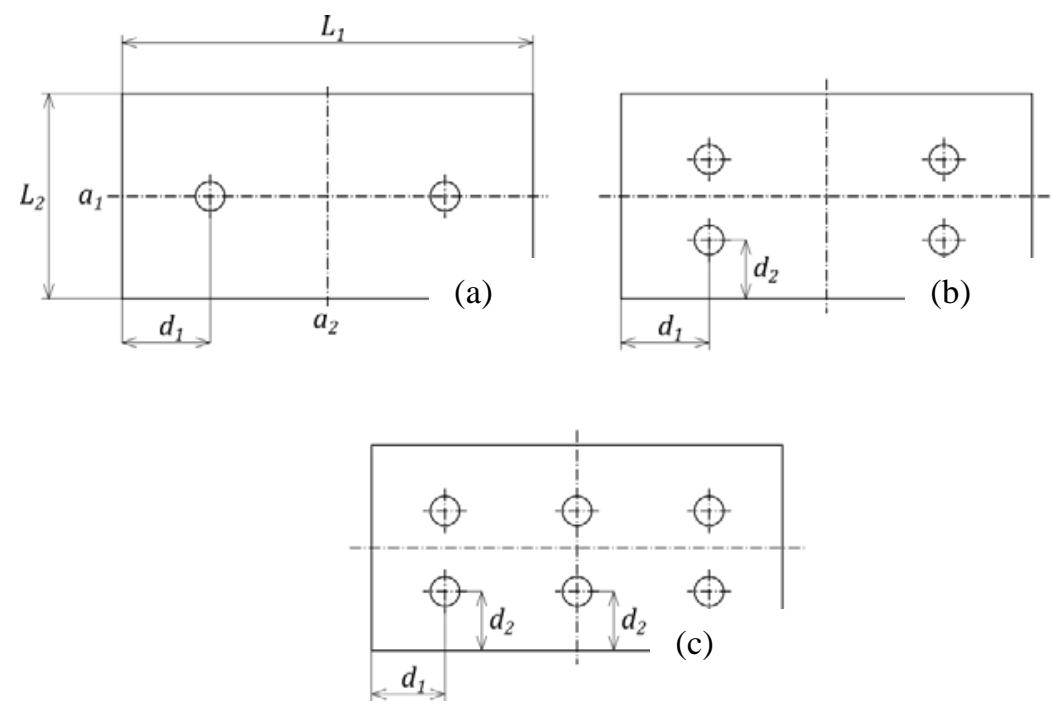

Fig. 6. The three considered suction cup patterns: (a) Pattern A; (b) Pattern B; (c) Pattern C.

The suction cup centers are positioned symmetrically with respect to the axes $a_{1}$ and $a_{2}$

\subsection{Slab properties}

The slab was modeled as a thin plate, and the material was considered isotropic and defined by the following parameters: 
- Young modulus E: $68 \mathrm{GPa}$

- Bulk density $\rho: 2380 \mathrm{Kg} / \mathrm{m}^{3}$

- Poisson ratio $v: 0.22$

The values were set according to Raimondo et al. (2010). The same paper reports that large slabs exhibit a limited sensitivity to the presence of microstructural defects (the coarsest pores are approximately $50 \mu \mathrm{m}$, and the critical defect size is in the $215-250 \mu \mathrm{m}$ range). This is an important remark because it permits taking a classical approach in evaluating the admissible stress, instead of an approach based on the fracture mechanics.

\subsection{Methodology}

Once the components and the pattern parameters were defined, it was possible to define a methodology to address the problem.

The aim of the investigation was set as: among the three considered, find the most suitable suction cup pattern and its optimal parameter values (Fig. 6) for each size and thickness considered.

To achieve this aim, the following two aspects were taken into account:

1) Maximum weight that the number of suction cups of the pattern can support.

2) Maximum stress and deformation on the slab during the lifting operation. The slab was considered lifted in a direction perpendicular to its plane.

For the first aspect, the maximum thickness th (in $\mathrm{m}$ ) that each suction cup pattern can support for each slab size was computed as:

$$
t h=\frac{n W_{s}}{\rho A}
$$

where $n$ is the number of suction cups of the considered pattern, $\rho$ the density (in $\mathrm{kg} / \mathrm{m}^{3}$ ) of the porcelain stoneware, $A$ the surface area of the slab (in $\mathrm{m}^{2}$ ), and $W_{\mathrm{s}}$ the maximum weight (in $\mathrm{kg}$ ) that a single suction cup can lift (set equal to $20 \mathrm{~kg}$ ). In Table 2 the results are summarized:

\begin{tabular}{|c|c|c|c|c|c|}
\hline \multirow{2}{*}{$\boldsymbol{n}$} & \multicolumn{5}{|c|}{ Size [mm] } \\
\cline { 2 - 6 } & $1200 \times 1200$ & $1600 \times 1600$ & $1200 \times 2400$ & $1000 \times 3000$ & $1600 \times 3200$ \\
\hline 2 & 11 & 6 & 5 & 5 & 3 \\
\hline 4 & 23 & 13 & 11 & 11 & 6 \\
\hline 6 & 35 & 19 & 17 & 16 & 9 \\
\hline
\end{tabular}

Table 2. Maximum thickness (rounded down) in mm for each combination of suction cups and slab sizes

For the second aspect, different sets of structural simulations were run on the finite element software ANSYS Workbench. Since the thickness of the slab is very small compared to its size, the slab was modeled as a 2D shell. According to Kovačević et al. (2008), this allows to reduce 
computational time without a substantial loss of accuracy. The following constraints and loads were considered on the slab:

- Fixed support rings (200 mm diameter) at the level of the contact region between the slab and the suction cups.

- Standard earth gravity (oriented normally to the surface of the slab).

- Inertial acceleration (positive if upward oriented, so that gravitational acceleration is negative).

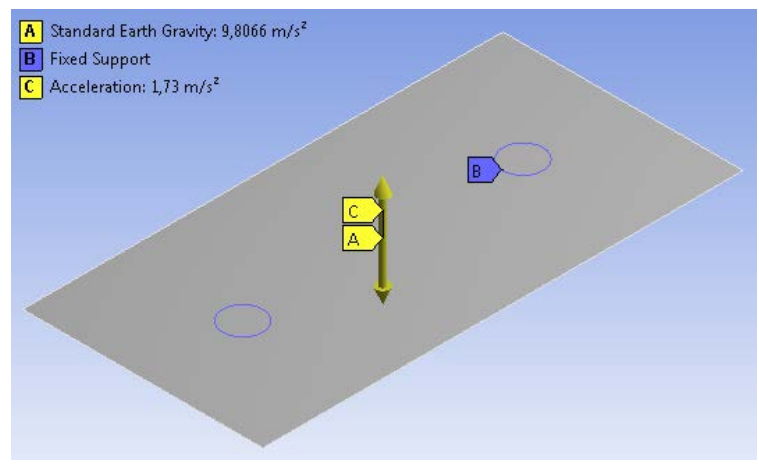

Fig. 7. Constraints and loads applied on the slab

The fact that suctions cups were not included into the simulations and their action was modeled with a fixed support is a simplification, since the introduction of a realistic model of suction cups (hyperelastic material, different pressure inside and outside, friction, etc.) would have caused an important rise in the model complexity, with no significant improvement of the results accuracy.

The lifting movement, usually carried out by manual operators, was modeled with a law of dynamic response of second order, whose shape can well approximate the human dynamic motion during the lifting operation (Ueno et al. 1996).

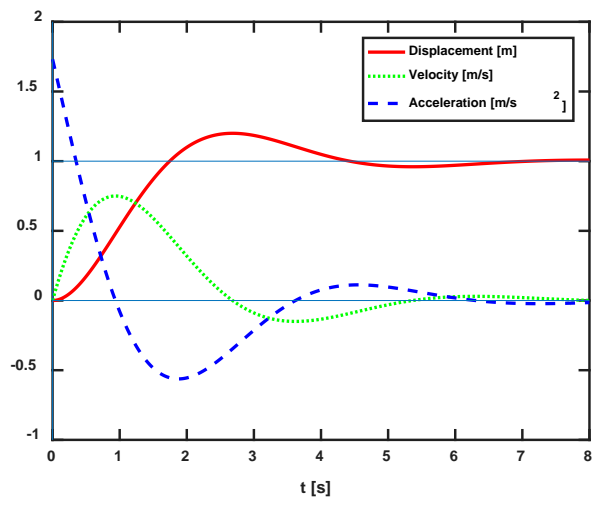

Fig. 8. A second order displacement law and its two derivatives 
The displacement law in Fig. 8 was computed by setting the overshoot to 20\%, the settling time to 5 seconds and the horizontal asymptote to 1 meter, values that were considered adequate if the slab is lifted by manual operators from the floor. The corresponding value of natural pulsation and damping factor are $\omega_{n}=1.316 \mathrm{rad} / \mathrm{s}$ and $\delta=0.456$, respectively. Only the positive values of the acceleration contribute to the increase of the stress values (being the inertial force added to the gravitational load), and since the material fatigue is not considered in this analysis, only the maximum positive acceleration is relevant. The maximum positive acceleration is at the initial time instant, and its value can be computed as $\omega_{n}{ }^{2}$. Therefore, the inertial acceleration considered in the static-structural simulation was $\omega_{n}^{2}=1.73 \mathrm{~m} / \mathrm{s}^{2}$.

\subsection{Meshing parameters}

A proper control of the meshing process is fundamental to achieve good accuracy and reliability of the results. In Fig. 9 the meshing approach is shown, with particular focus on the region in proximity of the fixed rings. In that region having the mesh denser is desirable, considering that in this zone the stress is supposed to be maximum.
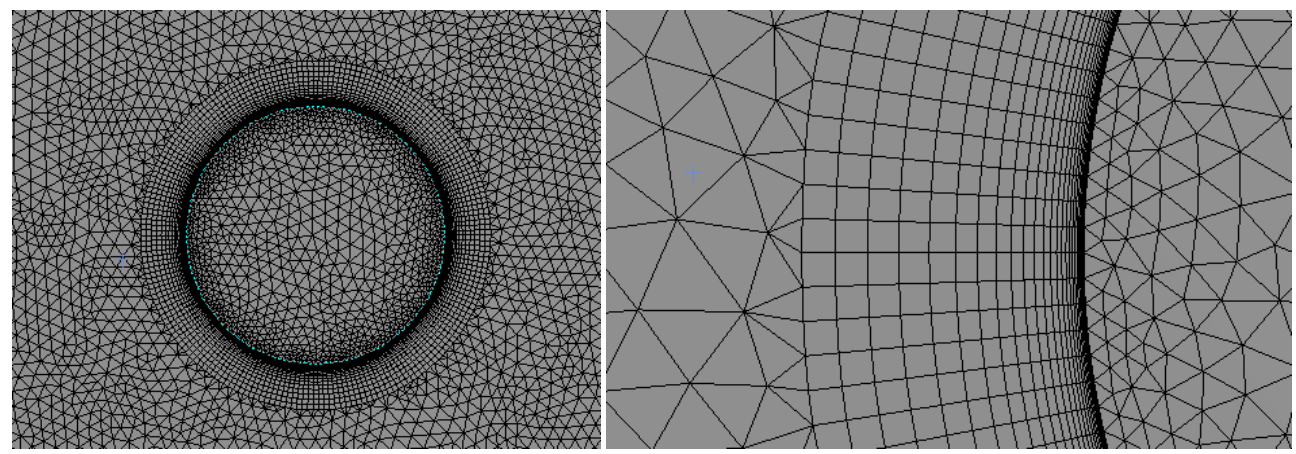

Fig. 9. Mesh used. At right, a close-up view of the mesh near a fixed ring

To obtain the mesh shown in Fig. 9, a triangles-based method was used, setting the following parameters:

- $\quad \min$ size: $1 \mathrm{~mm}$

- $\max$ face size: $10 \mathrm{~mm}$

The method was inflated near each ring in order to obtain smaller elements to the extent that the distance from ring decreases. The inflation properties were set in the following way:

- number of layers: 25

- growth rate: 1.2

- maximum thickness: $40 \mathrm{~mm}$

Furthermore, a sizing of 200 elements was added on each ring.

\subsection{Safety factor choice}

Before examining the stress distribution on the slabs, it is necessary to define the maximum stress admissible. For ceramic tiles, the modulus of rupture and the breaking strength have to be determined according to the BS EN ISO 10545-4, and the standard requirement for the modulus of rupture, according to ISO 13006 is $35 \mathrm{MPa}$. The admissible stress $\sigma_{a}$ (in MPa) was computed as:

$$
\sigma_{a}=\frac{35}{S F} \quad[\mathrm{MPa}]
$$


Where SF is the safety factor. The choice of $35 \mathrm{MPa}$ could be already considered a safe choice, since according to Raimondo et al. (2010) the modulus of rupture of large-sized ceramic slabs is around $70 \mathrm{MPa}$. However, there is a chance to find in the market large-sized ceramic slabs that have a lower modulus of rupture (down to $35 \mathrm{MPa}$, the lower value in compliance with the requirement). The safety factor $S F=1.75$ was chosen, and thus an admissible stress equal to $20 \mathrm{MPa}$. In addition, a limit of $4 \mathrm{~mm}$ in the admissible vertical elastic displacement was set (Raimondo et al. evaluated $5 \mathrm{~mm}$ the maximum elastic displacement).

\section{Results and discussion}

In this section, the results of the sets of static-structural simulations run in ANSYS Workbench are shown and analyzed.

\subsection{Investigation of the pattern parameters variation}

The first set of simulations was aimed at assessing the values of the parameters reported in Fig. 6 that ensure minimal deformations on the slabs. A parametrical analysis on a fixed thickness of $6 \mathrm{~mm}$ (the most common slab thickness) was performed for the different slab sizes. For size types 1, 2 and 3 (Table 1) only Pattern A and Pattern B were considered, whereas for size types 4 and 5 only Pattern B and Pattern C. For what concerns Pattern B, wherein two parameters are present, two different strategies were utilized, one for the square sizes and one for the rectangular ones:

- Square sizes: because of the symmetry, the center coordinate of the suction cup was varied along the slab diagonal, so that the investigation was only one-parameter dependent.

- Rectangular sizes: a 2D grid was defined and the vertical deformation (i.e. the elastic vertical displacement) evaluated on each grid point.

With regard to Pattern $C, d_{2}$ was fixed in the optimal position found for Pattern $B$, and only $d_{1}$ was varied. This strategy is admissible if the deformation in the midpoint (positioned on the axis $a_{1}$, Fig. 6) between two suction cups does not significantly vary when adding the two new suction cups (while maintaining other conditions unaltered). This assumption was subsequently verified, obtaining variations on the deformations of $0.001 \mathrm{~mm}$ for the $1000 \mathrm{x}$ $3000 \mathrm{~mm}$ size and $0.08 \mathrm{~mm}$ for the 1600 x $3200 \mathrm{~mm}$ size.

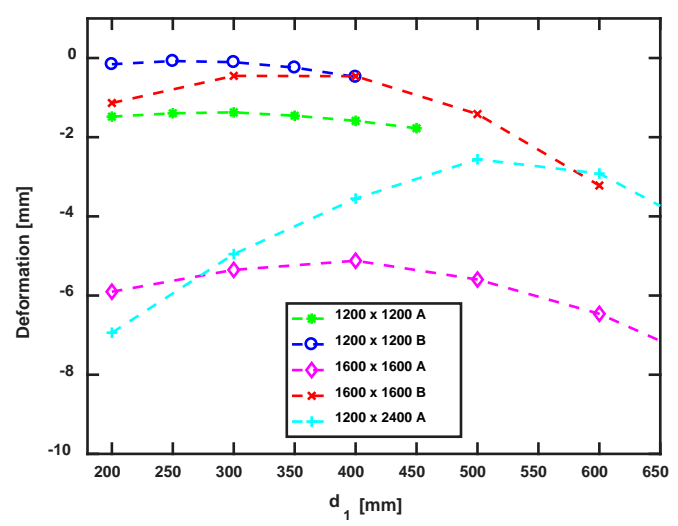

Fig. 10. One-parameter optimization for Pattern A and Pattern B, for different slab sizes 
In Fig. 10 the graphs obtained by the one-parameter dependent optimizations of Pattern $A$ and Pattern $B$ are shown. In the y-axis label, "Deformation” refers to the peak (negative) value of vertical displacement (i.e. orthogonal to the slab undeformed surface). In Figs. 11-14, the two-parameter optimization for Pattern B is shown for rectangular sizes: for the sake of clarity, the discrete sets of result values were interpolated with a surface. In Fig. 15, the one-parameter optimization for Pattern $C$ is shown. The parameters $d_{1}$ and $d_{2}$ were varied in general with 100 mm steps, with the exception of the $1200 \times 1200 \mathrm{~mm}$ slab case and the graphs of Fig. 15, where a $50 \mathrm{~mm}$ step was utilized for graphical purpose.

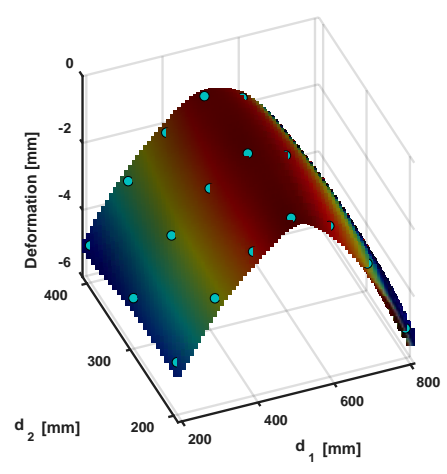

Fig. 11. 1200 x 2400 mm, Pattern B.

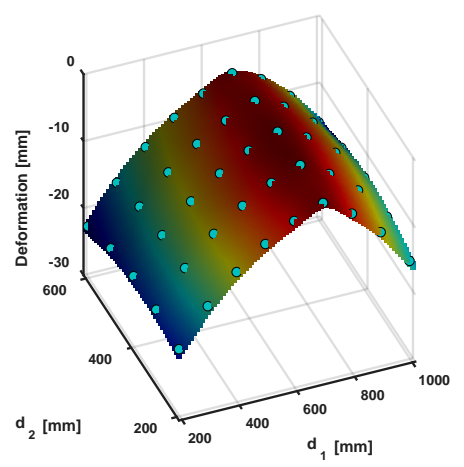

Fig. 13. 1600 x 3200 mm, Pattern B.

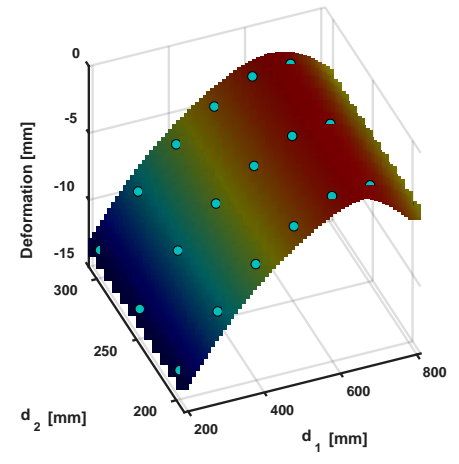

Fig. 12. 1000 x 3000 mm, Pattern $B$.

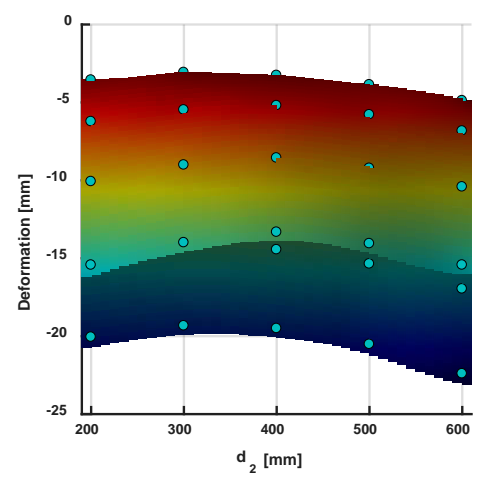

Fig. 14. $1600 \times 3200$ mm, Pattern $B$, different view.

By analyzing the Figs. 11-14, one can observe that the variation of $d_{1}$ affects pretty significantly the deformation, whereas the variation of $d_{2}$ has not an important effect, in fact the curve is fairly flat in respect to a $d_{2}$ variation. This can further support the one-parameter dependent strategy chosen for the variation investigation of the Pattern $C$ parameters. 


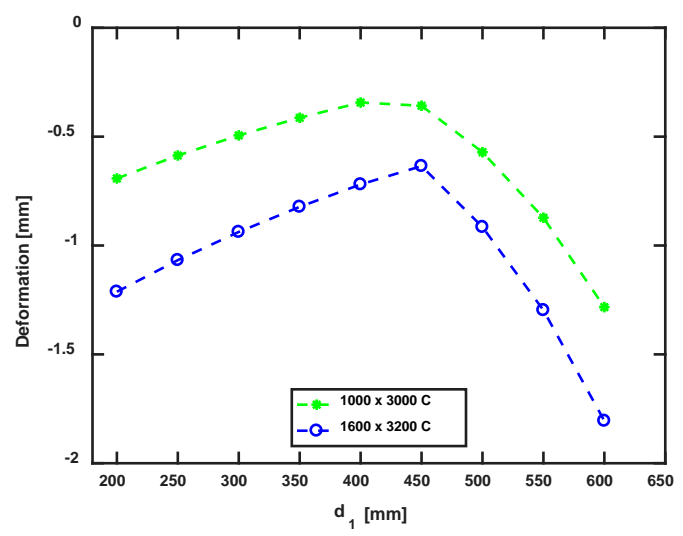

Fig. 15. One-parameter optimization for Pattern $C$

In Fig. 15, the marked change after the peak particularly visible in the 1600 x $3200 \mathrm{~mm}$ slab case is due to the fact that at about that point the maximum deformation does not occur anymore on an internal region of the slab, but on an edge.

In Table 3 the determined optimal values of the geometrical parameters are collected. The criterion that defines the optimum is the minimum peak value of the deformation.

\begin{tabular}{|c|c|c|c|c|c|}
\hline \multirow[b]{2}{*}{ Pattern } & \multicolumn{5}{|c|}{ Size [mm] } \\
\hline & $1200 \times 1200$ & $1600 \times 1600$ & $1200 \times 2400$ & $1000 \times 3000$ & $1600 \times 3200$ \\
\hline A & $d_{1} \approx 300$ & $d_{1} \approx 400$ & $d_{1} \approx 500$ & - & - \\
\hline \multirow{2}{*}{ B } & $d_{1} \approx 250$ & $d_{1} \approx 400$ & $d_{1} \approx 500$ & $d_{1} \approx 700$ & $d_{1} \approx 700$ \\
\hline & $d_{2} \approx 250$ & $d_{2} \approx 400$ & $d_{2} \approx 250$ & $d_{2} \approx 250$ & $d_{2} \approx 300$ \\
\hline \multirow{2}{*}{$\mathrm{C}$} & \multirow{2}{*}{ - } & \multirow{2}{*}{ - } & \multirow{2}{*}{ - } & $d_{1} \approx 400$ & $d_{1} \approx 450$ \\
\hline & & & & $d_{2} \approx 250$ & $d_{2} \approx 300$ \\
\hline
\end{tabular}

Table 3. Optimal values (in mm) of geometrical parameters for the different combinations of patterns and slabs sizes

The values of Table 3 are referred to a thickness of $6 \mathrm{~mm}$. The hypothesis that these values do not depend on the thickness in the range 3-15 mm was assumed and applied to the other sets of simulations illustrated in the next paragraphs. The suitability of this hypothesis can be (partially) verified by observing the graphs of Fig. 16, where four different thicknesses are taken into account for two different slab sizes (considering the Pattern A). By changing thicknesses, the value of $d_{1}$ corresponding to the peak remains the same. 


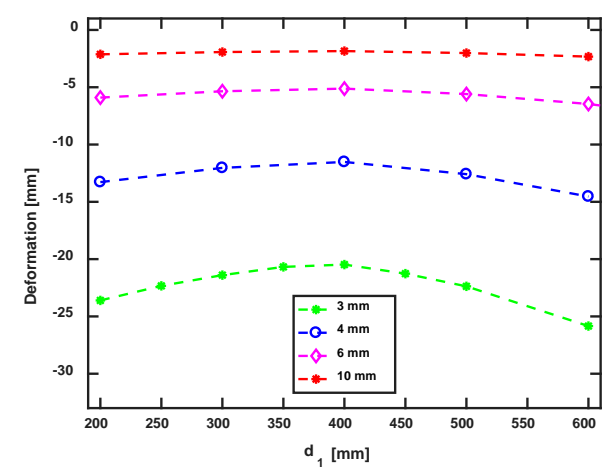

(a)

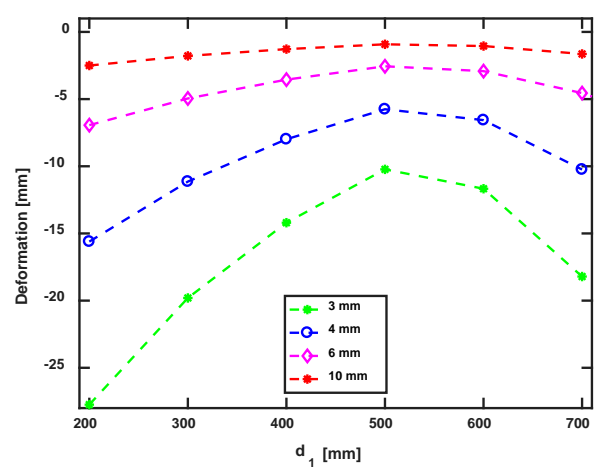

(b)

Fig. 16. Variation of $d_{1}$ (Pattern A) for four different thicknesses, for two different slab sizes:

(a) $1600 \times 1600$; (b) $1200 \times 2400$

\subsection{Thickness variation investigation}

The second set of simulations was run to assess the influence of the thickness variation, and to spot the thickness limit value that causes deformations higher than $4 \mathrm{~mm}$ and/or stresses higher than $20 \mathrm{MPa}$. To this aim, the pattern with the lower number of suction cups was considered for each size, and a parametrical analysis on the thickness variation was performed (the other parameters of Table 3 being fixed), leading to the graphs of Fig. 17. In the y-axis label, "Deformation" refers to the peak (negative) values of vertical displacement (i.e. orthogonal to the slab undeformed surface) and "Stress" refers to the maximum equivalent stress according to Von Mises criterion. The thickness was varied with $1 \mathrm{~mm}$ steps from 3 to $7 \mathrm{~mm}$ and with $2 \mathrm{~mm}$ steps from 7 to $15 \mathrm{~mm}$. The straight lines in Fig. 17 represent the maximum acceptable values of deformation (Fig. 17a) and stress (Fig. 17b).

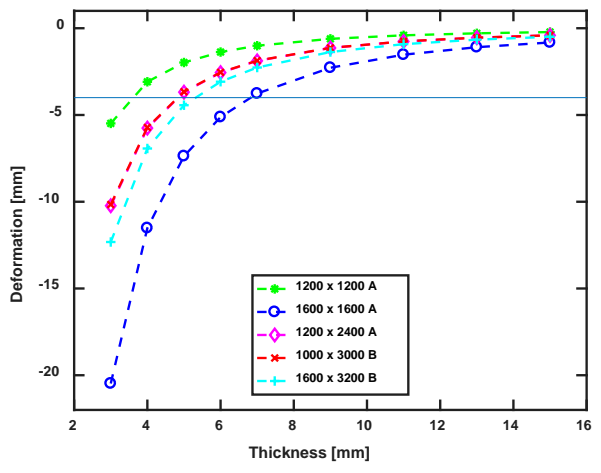

(a)

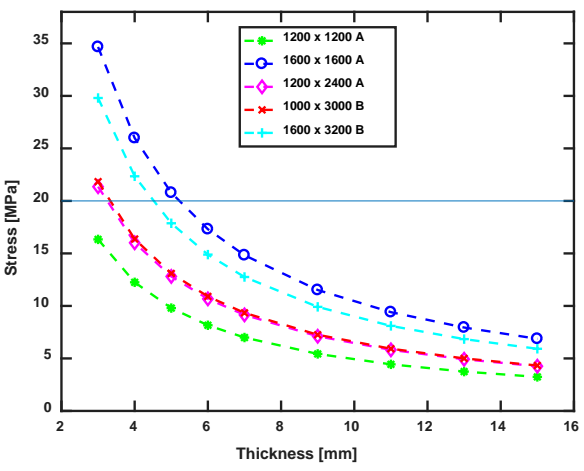

(b)

Fig. 17. (a) Deformation and (b) Stress as a function of thickness for the different combinations of sizes and patterns

By analyzing Fig. 17 one can observe that the condition on the stress allows lower thicknesses than the constraint on the deformation that proves stricter, and will be thus uniquely 
considered in the following. Table 4 summarized the results. The values represent the maximum allowed thickness to compel with the constraint of the maximum deformation value of $4 \mathrm{~mm}$.

\begin{tabular}{|c|c|c|}
\hline Pattern & $\begin{array}{c}\text { Size } \\
{[\mathrm{mm}]}\end{array}$ & $\begin{array}{c}\text { Thickness Limit } \\
{[\mathrm{mm}]}\end{array}$ \\
\hline A & $1200 \times 1200$ & 4 \\
\hline A & $1600 \times 1600$ & 7 \\
\hline A & $1200 \times 2400$ & 5 \\
\hline B & $1000 \times 3000$ & 5 \\
\hline B & $1600 \times 3200$ & 6 \\
\hline
\end{tabular}

Table 4. Thickness limit under which the threshold value of deformation of $4 \mathrm{~mm}$ is exceeded, for different combinations of sizes and patterns

\subsection{Additional analyses}

The values of Table 4 suggest that if the thickness is lower than the thickness limit the considered pattern is not suitable, thus a pattern with a higher number of suction cups is required. The last set of simulations was performed to asses if the pattern named with the very successive letter is able not to exceed the threshold values of deformation and stress. Since the most critical case occurs for the thickness of $3 \mathrm{~mm}$, one can just verify that the thresholds are respected for that case, because if it holds true, they are respected for all the thicknesses in the range. As it can be seen in Table 5, all the values are beyond the deformation and stress thresholds. Some of the complete stress and deformation maps are shown in Figs. 18-20.

\begin{tabular}{|c|c|c|c|}
\hline Pattern & $\begin{array}{c}\text { Size } \\
{[\mathrm{mm}]}\end{array}$ & $\begin{array}{c}\text { Stress } \\
{[\mathrm{MPa}]}\end{array}$ & $\begin{array}{c}\text { Deformation } \\
{[\mathrm{mm}]}\end{array}$ \\
\hline B & $1200 \times 1200$ & 3.12 & -0.3 \\
\hline B & $1600 \times 1600$ & 6.92 & -1.84 \\
\hline B & $1200 \times 2400$ & 15.19 & -3.85 \\
\hline C & $1000 \times 3000$ & 7.91 & -1.37 \\
\hline C & $1600 \times 3200$ & 11.16 & -2.54 \\
\hline
\end{tabular}

Table 5. Maximum equivalent stresses (Von Mises) and peak deformation values for different combinations of sizes and patterns, for the thickness of $3 \mathrm{~mm}$ 


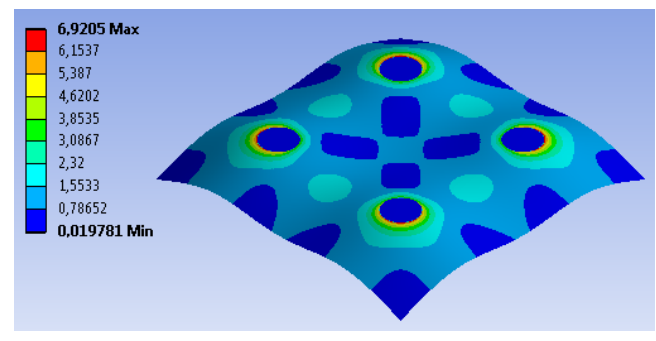

(a)

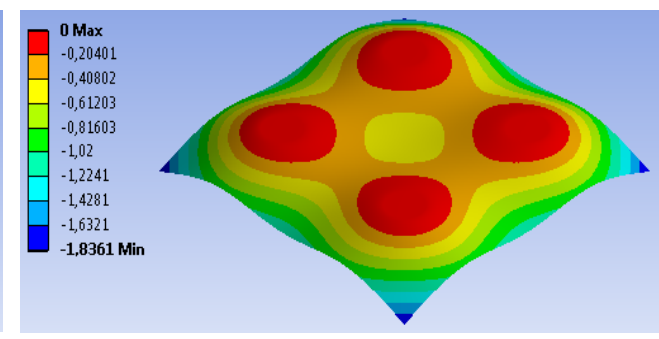

(b)

Fig. 18. 1600 x 1600 x 3 mm, Pattern B. (a) Equivalent stress in MPa; (b) Vertical deformation in $\mathrm{mm}$

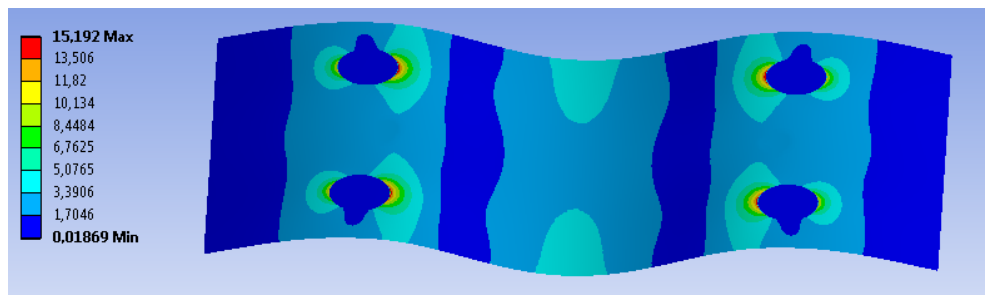

(a)

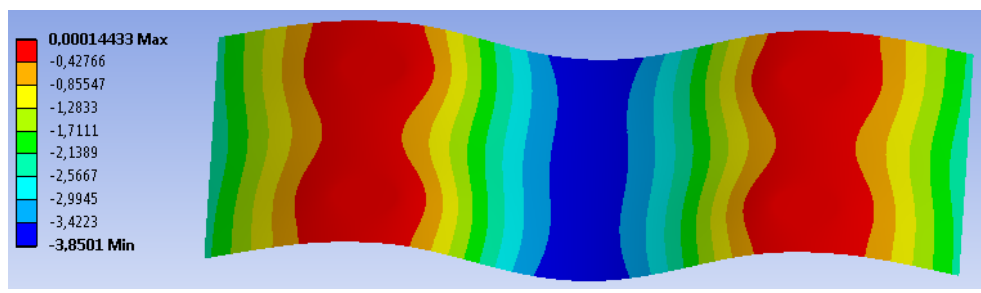

(b)

Fig. 19. 1200 x 2400 x 3 mm, Pattern B. (a) Equivalent stress in MPa; (b) Vertical deformation in $\mathrm{mm}$ 


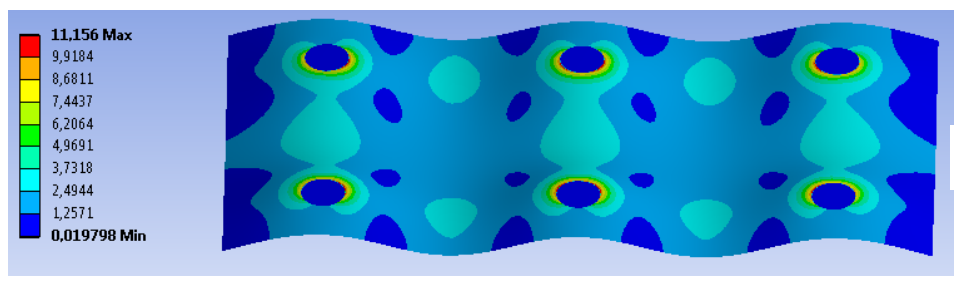

(a)

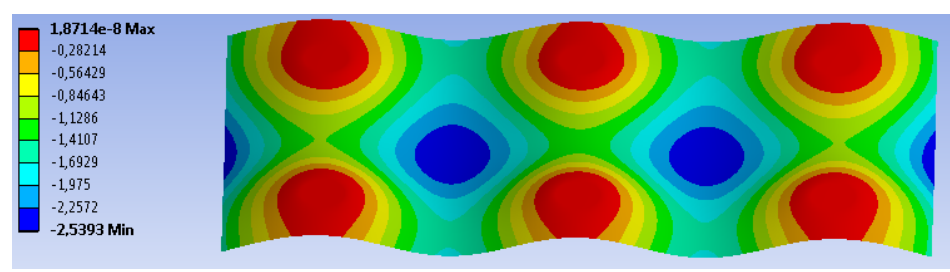

(b)

Fig. 20. 1600 x 3200 x 3 mm, Pattern C. (a) Equivalent stress in MPa; (b) Vertical deformation in $\mathrm{mm}$

By taking into account both the thickness limitations for the different patterns due to the suction cup force presented in Table 2 and the thickness limitations due to the deformation and stress thresholds presented in Table 4, the summarizing Table 6 was compiled. The results collected in this way can provide a useful guideline for selecting the type of suction cup pattern to use in specific cases. It has to be pointed out that the patterns reported as optimal in Table 6 are the simplest ones able to perform the task (e.g. if a task can be performed by the Pattern A, it can be performed by Pattern $B$ and Pattern $C$ as well, whereas vice versa does not hold). As for the 1600 x $3200 \mathrm{~mm}$ size, if the thickness overcomes $9 \mathrm{~mm}$ only 6 suction cups are not able to provide the proper gripping force, therefore the number of suctions cups has to rise.

\begin{tabular}{|c|c|c|c|c|c|c|c|c|c|c|c|c|}
\hline \multirow[b]{2}{*}{ Size $[\mathrm{cm}]$} & \multicolumn{11}{|c|}{ Thickness [mm] } & \\
\hline & & & & & & & & & & & & \\
\hline $120 \times 120$ & B & \multicolumn{7}{|c|}{ A } & \multicolumn{4}{|c|}{ B } \\
\hline $160 \times 160$ & \multicolumn{9}{|c|}{ B } & & & C \\
\hline $120 \times 240$ & \multicolumn{8}{|c|}{ B } & \multicolumn{4}{|c|}{$\mathrm{C}$} \\
\hline $100 \times 300$ & & & \multicolumn{6}{|c|}{ B } & \multicolumn{4}{|c|}{$\mathrm{C}$} \\
\hline $160 \times 320$ & \multicolumn{6}{|c|}{$\mathrm{C}$} & \multicolumn{6}{|c|}{ / } \\
\hline
\end{tabular}

Table 6. Optimal patterns suitable for each size and thickness 


\section{Conclusions}

Large-sized ceramic slabs with very low thickness (down to $3 \mathrm{~mm}$ ) have become common market products in the last decades, thanks to the outstanding improvements in materials and production cycles. The handling and transportation of these large slabs have to be carried out properly, in order to prevent high stresses and deformations that could damage the products. In the vast majority of cases, the handling relies on suction cups-based devices that are able to provide the necessary gripping force and are extremely suitable, since they only need contact with the upper slab face to operate. The number of suction cups and their corresponding arrangement is crucial for an optimal stress and deformation distribution on the slab. A methodology to address the issue was proposed and a numerical study relying on finite element analysis was conducted in order to investigate the effects of the variation of the pattern type and its geometrical parameters. Furthermore, a guideline aimed at supporting procedures for the selection of the suction cup pattern was provided, and it can be exploited to deal with the handling of the most common large-sized ceramic slabs on the market.

\section{Acknowledgements}

This research has been realized inside the project IPERCER with the aim of developing energy efficient solutions for the production of large porcelain stoneware tiles. The authors acknowledge the support of the European Union and the Region Emilia-Romagna (inside the POR-FESR 2014-2020, Axis 1, Research and Innovation).

\section{References}

ANSYS Workbench user’s guide, release 15.0, November 2013.

Brevetti Montolit, Professional tools for tile layers, General product portfolio (2016).

CAESAR ceramics, Project Evolution catalogue (2015).

Ceramic tiles -- Definitions, classification, characteristics and marking, ISO 13006 (2012).

Ceramic tiles -- Determination of modulus of rupture and breaking strength, BS EN ISO 105454 (2014).

Fragassa C (2015). Limits in Application of International Standards to Innovative Ceramic Solutions, International Journal for Quality Research, 9, 2, 279-298.

Fragassa C (2016). Modelling the viscoelastic response of ceramic materials by commercial Finite Elements codes. FME Transactions, 44, 1, pp 58-64.

Fragassa C (2016). Flexural Testing Machine as an Off-Line Control System for Quality Monitoring in the Production of Bended Ceramic Tiles. International Journal for Quality Research, Vol. 10 - N. 2: pp 373-388.

Fragassa C, Pavlovic A, Ubertini F (2014). Experimental evaluation of pyroclastic deformation on mechanical properties of grès porcelain stoneware, IMK 14 - Research \& Development in Heavy Machinery 20 3, EN77-85.

Gozzi A, Marani F, Dondi M, Raimondo M, Zabelli C (2009). Technological behaviour of lowthickness ceramic tile, Ceramic World Review 83, 78-82.

Kovačević D, Matijević, Rašeta A (2008). An optimal numerical model for nonlinear behavior of reinforced concrete frames. JSSCM, Vol. 2, No. 1, pp. 100-120.

Leonelli C, Bondioli F, Veronesi P, Romagnoli M, Manfredini T, Pellacani G, Cannillo V (2001). Enhancing the mechanical properties of porcelain stoneware tiles: a microstructural approach, J. Eur. Ceram. Soc. 21, 785-793. 
Li R, Tian Y, Wang P, Shi Y, Wang B (2016). New analytic free vibration solutions of rectangular thin plates resting on multiple points supports, International Journal of Mechanical Sciences 110, 53-61.

Morselli U (2009). Plastic state shaping with variable thicknesses, Ceramic World Review 83, 96-100.

Pavlovic A, Fragassa C (2016) Modelling the Viscoelasticity of Ceramic Tiles by Finite Element. Proceedings of the 8th Conference on Times of Polymers \& Composites. American Institute of Physics (AIP). 19-23th June 2016, Ischia (Italy). Vol. 1736, No. 4949749; doi: 10.1063/1.4949749.

Pavlovic A, Ubertini F (2015). Equipment qualification in testing the flexural resistance of bended ceramic tiles, FME transactions 44, 146-153.

Raimondo M, Dondi M, Zanelli C, Guarini G, Gozzi A, Marani F, Fossa L (2010). Processing and properties of large-sized ceramic slabs, Bol. Soc. Esp. Ceram. V.49, 4, 289-296.

Raimondo M, Zanelli C, Guarini G, Dondi M, Marani F, Fossa L (2009). Innovative porcelain stoneware slabs (Lamina) from processing to applications, Proceedings of 11th International Conference on Advanced Materials, ICAM 2009, Rio de Janeiro (Brazil), September 20-25, 78-83.

Sánchez E, García-Ten, Sanz V, Moreno A. (2010). Porcelain tile: Almost 30 years of steady scientific-technological evolution, Ceramics International 36, 831-845.

Tasdemerici A, Hall W (2009). Development of novel multilayer materials for impact applications. A combined numerical and experimental approach, Materials and design 5, 1533-1541.

Timoshenko S, Woinowsy-Krieger S (1989). Theory of Plates and Shells, Mc Graw-Hill, second edition.

Tsamasphyros G, Bikakis GS (2009). Finite element modeling and analytical simulation of circular GLARE fiber-metal laminates subjected to lateral indentation, JSSCM, Vol. 3, No. 2, pp. 67-80.

Tucci A, Esposito L, Malmusi L, Rambaldi E (2007). New body mixes for porcelain stoneware tiles with improved mechanical characteristics, J. Eur. Ceram. Soc. 27, 1875-1881.

Ueno T, Sunaga N, Brown K, Asada H (1996). Mechanism and control of a dynamic lifting robot, Proceedings of the 13th ISARC, pages 95-102.

Vivona D, Piccinini F (2009). Low thickness for large eco-efficient size, Ceramic World Review 83, 90-95.

Wang ZW, Tang CY, Tsui CP, Gao B (2008). Optimization design for graded porous tubular structures. JSSCM, Vol. 2, No. 2, pp. 80-90. 